\title{
The Progression of Multi-Dimensional Water Column Analysis in a Processing Environment
}

\author{
Corey M. COLLINS, P.Eng, Canada \\ Topic C: innovations in processing techniques

\section{INTRODUCTION}

With the ever-increasing availability of CPU processing power and data storage, multibeam sonar systems used for shallow water surveys are now capable of collecting full water column imagery data. This imagery is being used by post processing software as a way to supplement bottom detection results, often for the purpose of detecting the shoalest parts of obstruction such as ship wrecks.

This paper will describe how water column imagery can be interrogated as part of an integrated bathymetric processing workflow. It will highlight tools and techniques that can be used in conjunction with standard swath analysis for the purposes of least depth detection. Perhaps more interestingly it will also describe how water column imagery can be represented in a 3D environment using the latest point cloud processing and visualization techniques. By representing water column imagery as a point cloud it becomes much easier to determine, shape, extent and volume of features in the water column.

Further to the reading and display of the water column data, the ability to interrogate water column data in an effort to add it as a supplement to existing bathymetric data will be described. The ability to incorporate post-processed data such as TrueHeave or SBET information from POSPac as well as the ability to compute total propagated uncertainty using real-time uncertainties allows the water column data to be fully and robustly integrated into existing bathymetric processing workflows.

\section{WATER COLUMN IMAGING}

Even though water column imaging was originally developed primarily for fisheries, today's technical and financial pressures to extract as much information as possible from hydrographic data makes water column data an obvious fit in the industry. Access to this data in post processing software provides significant advantages for hydrographic data quality control [Hughes Clarke et. al., 2006].

With the release of HIPS 7.1.1, the reading and the displaying of water column Imagery was added. Initial support for $\mathrm{WCl}$ includes Kongsberg (.all, .wcd) sonars and Reson 7K (.s7k) sonars. The data can be read and displayed as a curtain image in Swath Editor, as well as read and displayed as a 3D point cloud in Subset Editor. The data as displayed can then be used to help aid the Processor in making decisions with regards to the bathymetric data. Whether it is the ability to verify valid or invalid data or the ability to identify areas of additional interest in regards to further investigation or even re-survey [Collins, 2012]. As development has progressed with the $\mathrm{WCl}$ implementation and with the release of HIPS 8.0, the user is now able to utilize the WCI as a supplement to traditional bathymetry. 


\section{CARIS HIPS}

With a proven reputation of being a comprehensive bathymetric data cleaning and validation tool, it is only fitting that CARIS HIPS allow users to read, visualize, and utilize WCI data in a multidimensional environment. With proven automated data cleaning filters and algorithms, which assist in today's high data volume environments, $\mathrm{WCl}$ is a great addition to the work flows currently available in the application.

When first approaching this initiative, the goal was to implement Water Column Imaging within HIPS is such a way that it would be useful for a variety of applications. Examples include, quality control of bottom detections, least depth determination, 3D visualisation of water column data, volume calculations, environmental monitoring, detecting gas seeps, etc. Due to the size and complexity of the development effort, the initiative was broken down into five phases; 1) read WCI data, 2) display WCI data in Swath Editor, 3) display WCI data in Subset Editor, 4) ray-trace water column data, and 5) additional processing of WCI data [Collins, 2012]. With the release of version 7.1 .1 in early 2012, users have seen the implementation of the first 3 phases. As the $\mathrm{WCl}$ initiative progresses, phase four will be released in version 8.0 late 2012. Phases five will be tackled post version 8.0 and released to users as they become available.

\section{Swath Editor}

Over the last five to ten years we have seen a decrease in the line by line editing techniques and a dramatic increase in the directed editing approach within post processing software. However, there is still value in having access to a line by line editor, such as the Swath Editor in an effort to QC and troubleshoot the data when issues arise. With that said the ability to view the $\mathrm{WCl}$ data in a $2 \mathrm{D}$ line by line editor is seen as beneficial and is a great addition for quality control and detailed investigation [Collins, 2012]. In order for the WCI data to be viewed in Swath Editor, the data as recorded in the raw data file has to be mapped into a two-dimensional near-vertical plane with respect to the transducer. This involves transforming the data from polar to Cartesian coordinates [Hughes Clarke, 2006].

With the ability to read the $\mathrm{WCl}$ and the transforming of the data to Cartesian coordinates, Swath Editor now has the ability to display individual polar plots (across track image) of WCI data associated with a specific profile as well along track image associated with the nadir beam. The user is able to step through these $\mathrm{WCl}$ images, in the Across Track, on a profile by profile basis. The view can also be stacked where all images are stacked one on top of the other with the highest intensity value showing through. This option provides a representation of all WCI data within the Plan view as opposed to one profile at a time. The Along Track view can be stacked as well, where all beams within a profile are stacked one on top of the other with the highest intensity value showing through.

These views can be utilized to validate what is being presented in the bathymetry. To add to these views, the bottom detections can be displayed, which allows for easy comparison of how the bathymetry relates to what is present in the water column imagery. This could potentially highlight what the sonar missed in the water column, as well as aid the decision of whether the bathymetry is valid or not. Should the need arise, where there is important information within the 
$\mathrm{WCl}$ data that is not present in the bathymetry, the user has the ability to select the desired $\mathrm{WCI}$ information and add it as additional bathymetry as seen in Figure 1.

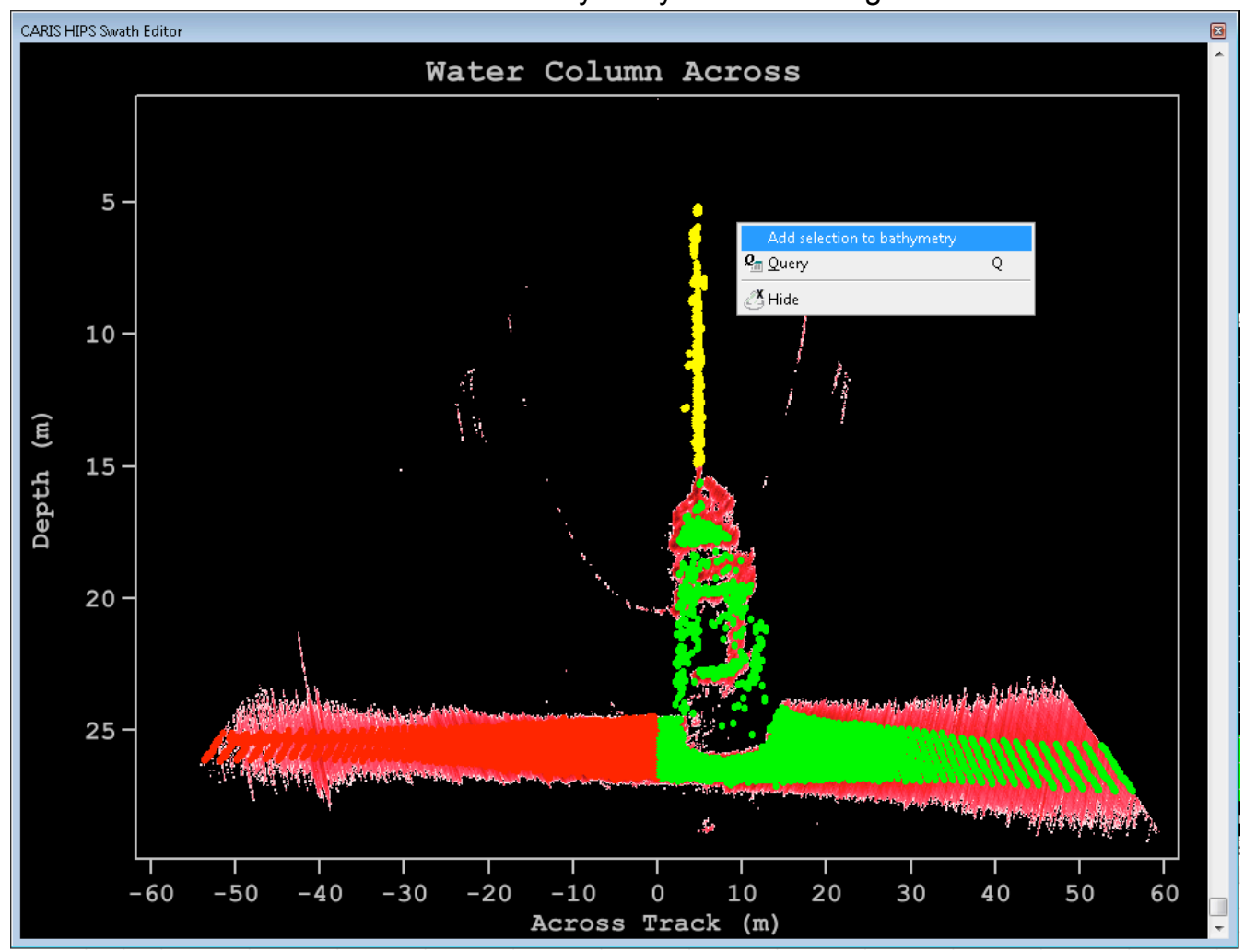

Figure 1: A selection of water column data in Swath Editor to be added as additional bathymetry.

All existing options and functionality within the current Swath Editor, where relevant, have been connected with the $\mathrm{WCl}$ views to keep work flows consistent and familiar. Functionality such as colouring, selection of bottom detections, querying of bottom detections, editing of bottom detections as well as editing the additional bathymetry can be carried out in both the WCI views. Furthermore, all views update to indicate changes made in other views.

\section{Subset Editor}

With the shift of data editing from a line by line approach to an area based directed editing approach; it was deemed critical that the WCI data could also be displayed in 3D space with the bathymetry. The Subset Editor, like the Swath Editor, also has the ability to load and display Water Column data, but in this case, as a geographically referenced 3D point cloud. Since CARIS has implemented a sophisticated 3D point cloud data structure that can store billions of multiattributed 3D points, a perfect container for the $\mathrm{WCI}$ data in 3D space already existed. This technology has been leveraged to load and display the water column data seamlessly into this area based editor [Masry et. al., 2009].

The key to displaying the data in $3 \mathrm{D}$ space is to translate the water column data from a vessel referenced $2 \mathrm{D}$ plot to a 3D geographically referenced point cloud. This has been done by taking the $\mathrm{WCI}$ data and automatically pushing it through the standard processing workflow (vertical adjustment, svc and merge) to reference the data geographically with respect to the same 
coordinate system as the bathymetry. Like the swath Editor, should the need arise, if there is important information within the $\mathrm{WCl}$ data that is not present in the bathymetry, the user has the ability to select the desired $\mathrm{WCl}$ information and add it as additional bathymetry as seen in Figure 2.

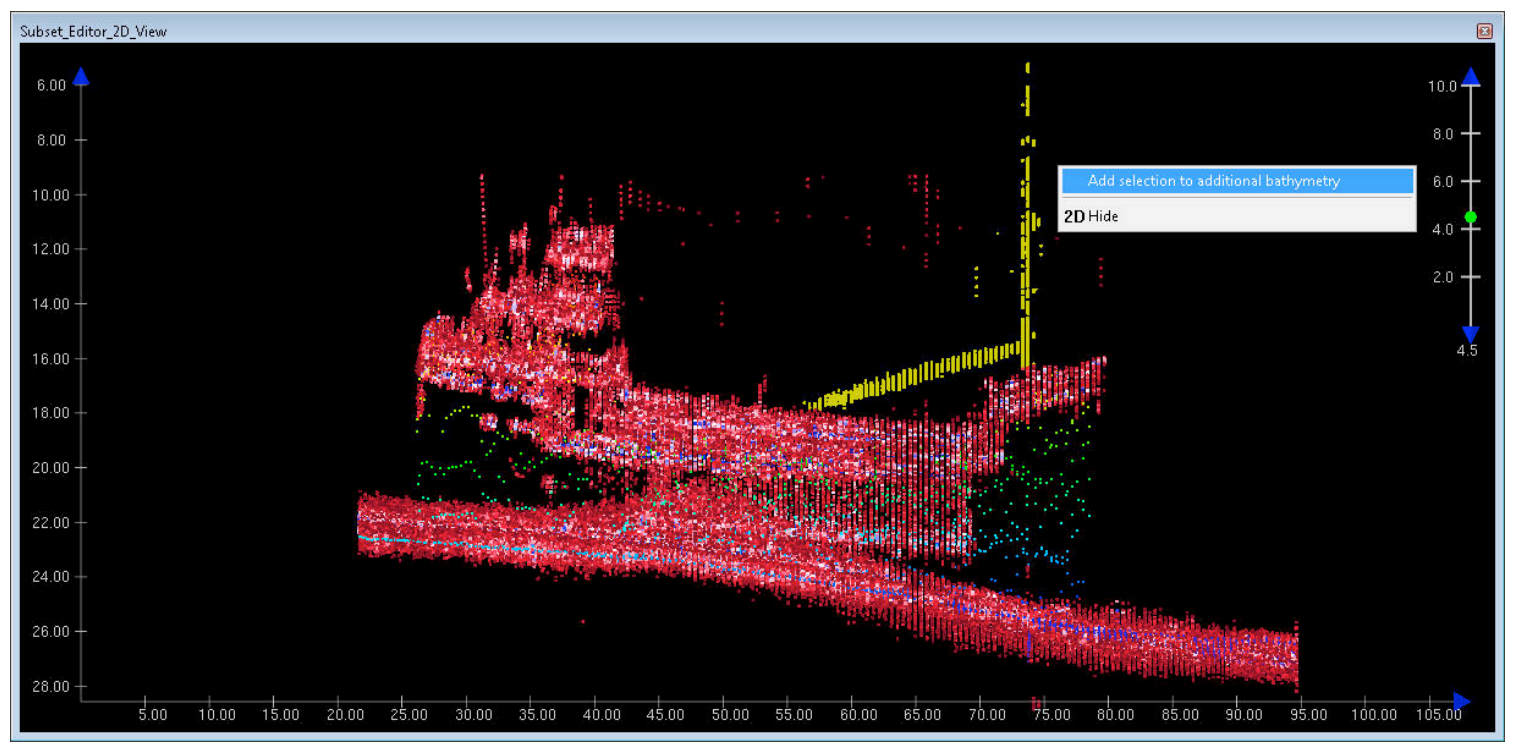

Figure 2: User can now interrogate water column data in Subset Editor and add it as additional bathymetry.

All existing options and functionality within the current Subset Editor, where relevant, have been connected with the $\mathrm{WCl}$ display to keep workflows consistent and familiar. Functionality such as colouring, point size, etc. can be used to alter the display of the bathymetry as well as the $\mathrm{WCl}$ data coupled with the ability to edit the additional bathymetry.

\section{ADDITIONAL BATHYMETRY}

In order to allow the user to utilize the water column information as data with position and depth, it was decided to leverage again the CSAR point cloud. What the user can expect to see here is an additional data source contained within the HIPS project labeled as additionalBathymetry as seen in Figure 3. With the desired information within the water column isolated and added to the additional bathymetry layer from either the Swath or Subset Editor, the user now has the ability to utilize this in the existing workflows. Once the user has decided that the information is of relevance and is required for a more complete representation of the submarine landscape, the data becomes available for things such as editing, uncertainty computation and surface creation.

As with the standard bathymetric workflow that currently exists in HIPS, the water column data that has been added to the additional bathymetry layer will be processed in the same way. Once the water column data has been selected and added to the additional bathymetry, the same processing options that have been applied to the bathymetry will be applied to ensure a properly geo-referenced point cloud of $\mathrm{WCI}$ data. These processes include proven ray tracing techniques (i.e. cone-cone intersection, Kongsberg supplied SVC dII) utilizing dynamic attitude data or postprocessed attitude information such as a TrueHeave or SBET solution from POSPac. It also 
includes vertical adjustments such as traditional tide or GPS tide as well as positioning the data utilizing standard as well a post-processed gyro and navigation data.

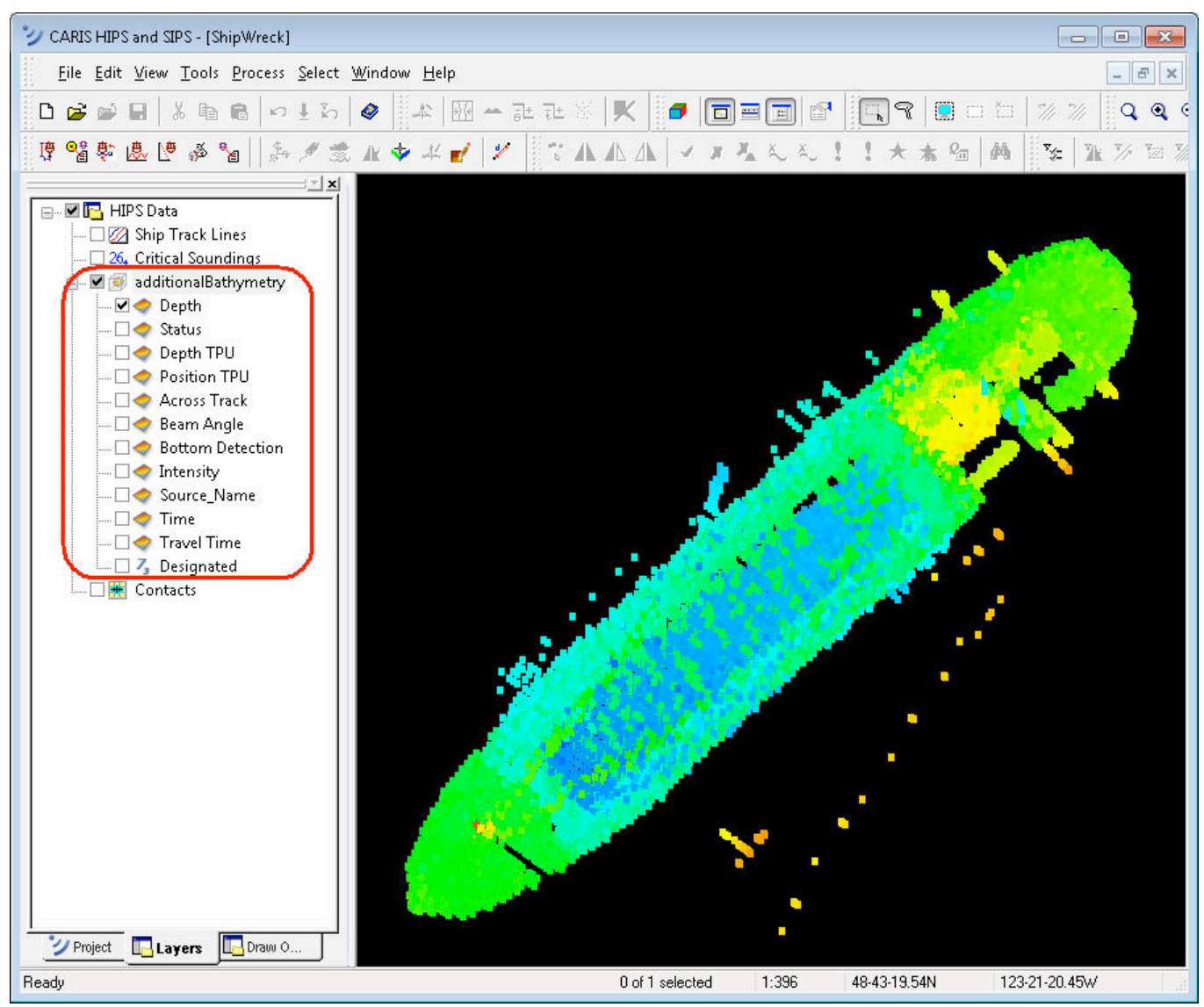

Figure 3: Additionally bathymetry layer leverages the CSAR point cloud technology to allow the $\mathrm{WCl}$ data to be utilized in standard processing workflows.

Once the $\mathrm{WCl}$ information has been properly geo-referenced as a point cloud it then becomes available for further processing such as your standard editing (accept, reject, designate, etc.) in the bathymetric editors. Furthermore, if desired, uncertainty information can be computed for the additional bathymetry and in turn utilized in surface creation such as CUBE. With the progression of the implementation of water column processing in a multi dimensional environment it can now be seen how water column data can be fully and robustly integrated into existing bathymetric processing workflows.

\section{CONCLUSION}

With the use of WCI by post processing software such as CARIS HIPS, it is apparent that this data can be used in a way to supplement bottom detection results. As this paper describes, the incorporation of water column data with bathymetry also provides a more complete representation of the submarine landscape as a whole and as such has the potential to allow multibeam data to be used for many non-traditional applications. 
This paper has also described how water column imagery can be interrogated as part of an integrated bathymetric processing workflow in CARIS HIPS. It also describes tools and techniques that can be used in conjunction with standard swath analysis for the purposes of visualization and quality control. It has also been shown how water column imagery can be represented in a 3D environment by using the latest point cloud processing and visualization techniques. Further to this it has been shown how the progression of this initiative in a multi dimensional environment has allowed the water column data to be fully integrated into existing bathymetric processing workflows utilizing the same processing options as applied to the standard bathymetry.

\section{REFERENCES}

Collins, C. (2012). "Multi-Dimensional Water Column Analysis as a Catalyst for Sustainable Hydrographic Survey." Presented at Shallow Survey Conference, 2012.

Hughes Clarke, J.E., Lamplugh, M., Czotter, K. (2006), "Multibeam Water Column Imaging: Improved Wreck Least-Depth Determination." Presented at Canadian hydrographic Conference 2006.

Hughes Clarke, J.E. (2006), "Applications of Multibeam Water Column Imaging for Hydrographic Survey." The Hydrographic Journal April, 2006.

Kongsberg, EM2040 Dataset (2011), Data provided courtesy of Canadian Hydrographic Service.

Kongsberg, EM3002 Dataset (2006), Data provided courtesy of John Hughes Clarke, OMG UNB, Canada.

Masry, M., Schwartzberg, P. (2009), "Marine High Density Data Management and Visualization." Presented at the seminar for Management of massive point cloud data: wet and dry. November 26, 2009.

Shallow Water Common Dataset (2012), Data collected for the 2012 Shallow Water Conference, February 2012.

\section{BIOGRAPHY}

Corey M. COLLINS, P.Eng. CARIS HIPSISIPSINotebook Product Manager. Received a Bachelor of Science in Engineering from the University of New Brunswick's Geodesy and Geomatics Program. Before joining CARIS he worked in Hydrography as well as Marine Geophysical Surveying. Professional Engineer with the Association of Professional Engineers and Geoscientists of New Brunswick (APEGNB), Canada.

Corey M. COLLINS

CARIS

115 Waggoners Lane

Fredericton

CANADA

Tel.: +1-506-458-8533

Fax: +1-506-459-3849

Email: corey.collins@caris.com

Web site: www.caris.com

Linkedln account: http://www.linkedin.com/groups?mostPopular=\&gid=3217878 
Facebook account: http://www.facebook.com/pages/CARIS-The-Marine-GIS-

Experts/123907500987669

Twitter account: twitter.com/CARIS_GIS 